\title{
WNEREFACFETECNOLOGGCA
}

\section{COMPARATIVO ENTRE FRAMEWORKS DE CSS BOOTSTRAP E BULMA PARA DESENVOLVIMENTO DE PROJETOS WEB}

\author{
COMPARATIVE BETWWEN CSS FRAMEWORKS BOOTSTRAP AND BULMA FOR
}

\section{WEB PROJECT DEVELOPMENT}

\author{
Leonardo Patrocinio Souza - leopatrocinioo@gmail.com \\ Felipe do Espirito Santo - felipe.santo@fatectq.edu.br
}

Faculdade de Tecnologia de Taquaritinga (Fatec) - Taquaritinga - São Paulo - Brasil

DOI: 10.31510/infa.v17i1.785

\section{RESUMO}

O desenvolvimento web está sempre em constante progresso: desenvolvedores criam novas ferramentas para auxiliar no processo de construção de projetos para a web e são inúmeras as ferramentas, com as mais diversas finalidades. Esse artigo tem como objetivo abordar os frameworks de CSS Bootstrap e Bulma, ferramentas que auxiliam no desenvolvimento de projetos web de forma geral. Observando a tecnologia atual, é muito importante que o desenvolvedor análise alguns pontos sobre esse processo de desenvolvimento, como, por exemplo, segurança, acessibilidade, responsividade e, principalmente, a experiência que o usuário terá em utilizar essa aplicação. Responsividade é um requisito de extrema importância. Ter uma aplicação que funcione bem em qualquer dispositivo móvel (celulares, tablets, dentre outros) é fundamental, pois torna o acesso mais fácil nesses dispositivos, fazendo com que o usuário localize de forma rápida suas buscas. Com base em uma pesquisa bibliográfica para apresentar os conceitos de frameworks e analisar as duas opções propostas, foi constatado que existem várias características diferentes em cada uma, porém o artigo ressaltou a introdução ao conceito, as semelhanças, os principais recursos, pontos fortes e fracos. Ambos os frameworks apresentaram vantagens e desvantagens e podem ser bons em diferentes aplicações web, mas em determinados pontos os estudos demonstraram as razões de escolher um deles.

Palavras-chave: Frameworks de CSS. Bootstrap. Bulma. Responsividade.

\begin{abstract}
Web development is always in constant progress: developers create new tools to assist in the process of building projects for the web and there are countless tools and the most diverse purposes. This article aims to approach the CSS frameworks Bootstrap and Bulma, tools that help in the development of web projects in general. Observing the current technology, it is very important that the developer analyzes some points about this development process, such as, security, accessibility, responsiveness and, mainly, the experience that the user will have in using this application. Responsiveness is an extremely important requirement. Having an application that works well on any mobile device (cell phones, tablets, among others) is essential, as it makes access easier on these devices, making the user quickly locate their searches. Based on a bibliographic search to present the concepts of frameworks and analyze the two proposed options, it was found that there are several different characteristics in each one, however, the article highlighted the introduction to the concept, the similarities, the main
\end{abstract}




\section{WTEERFAET TECNOLOGGCA}

resources, strengths and weaknesses. Both frameworks showed advantages and disadvantages and can be good in different web applications, but in certain points, the study demonstrated the reasons for choosing one of them.

Keywords: Frameworks de CSS. Bootstrap. Bulma. Responsiveness.

\section{INTRODUÇÃO}

Com o frequente avanço tecnológico, surgem dispositivos no mercado com acesso a sites e aplicativos. Com isso, desenvolvedores buscam constantemente pelo conhecimento e aprimoramento de novas técnicas, pois "os sites precisam se adaptar ao dispositivo utilizado pelo usuário" (SILVA,2014, p.1).

Para que o usuário tenha uma boa experiência com a aplicação que está sendo utilizada no dispositivo, o desenvolvedor pode fazer o uso de alguns frameworks, que são ferramentas que auxiliam no processo de desenvolvimento em uma aplicação web.

Existem diversos frameworks para desenvolvimento de aplicações, porém o foco será em frameworks para construção de estilos para os sites. Questionamentos podem surgir em relação se um framework é ou não uma biblioteca. Framework é um conjunto de bibliotecas ou componentes que são utilizados na criação da base onde a aplicação será desenvolvida. Com isso, baseado em uma pesquisa bibliográfica feitas em livros, sites e artigos científicos, o objetivo do artigo é comparar, analisar e ressaltar as vantagens e desvantagens de dois frameworks, Bootstrap em sua última versão 4.4.3 e o Bulma.

Desenvolver uma aplicação web do zero é uma tarefa bastante árdua e complexa. Ao invés de começar um projeto do zero, é útil e viável fazer o uso de frameworks que possam auxiliar no desenvolvimento. As opções disponíveis são várias, e o desenvolvedor pode se ver em qual framework melhor atenderia a necessidade para tal projeto (CONFIGR, 2019).

Este artigo visa apresentar as principais características do Bootstrap e do Bulma, seus pontos fortes e fracos, podendo assim auxiliar o desenvolvedor em relação a escolha entre os dois frameworks para um projeto de desenvolvimento para web.

\section{FUNDAMENTAÇÃO TEÓRICA}

O tamanho das telas dos dispositivos também à influência no desenvolvimento de sites, os conteúdos devem se adaptar a essas diferentes telas, o princípio que norteia o 
desenvolvimento das técnicas para adaptar o site ao dispositivo é conhecido como Design Responsivo (SILVA, 2014).

Atualmente existem várias técnicas que facilitam o desenvolvimento de sites responsivos, uma delas é utilizar frameworks (JAIN, 2014).

Frameworks quando estamos lidando com desenvolvimento de software podem ser considerados abstrações que unem vários projetos com uma funcionalidade em comum, o framework pode ter uma finalidade específica, por configuração, durante o desenvolvimento de uma aplicação (MULLER, 2008).

Os frameworks são um recurso cada vez mais importante no desenvolvimento de aplicações, estão presentes em praticamente todas as linguagens de programação, um framework normalmente oferece recursos que facilitam o desenvolvimento ao abstrair conceitos, normalmente de mais baixo nível, viabilizam o reuso de código (DEVMEDIA, 2018).

\subsection{Design responsivo}

O Termo Responsive Web Design surgiu em 2010, onde Marcotte afirma em seu artigo publicado ao site "A List A part" que "Responsive web design é a abordagem que sugere que o design e desenvolvimento devem responder ao comportamento do usuário e do ambiente baseando-se no tamanho da tela, plataforma e orientação" (MARCOTTE, 2010).

Segundo Arty (2015) o artigo de Marcotte:

Abordava a mudança na forma de uso da web com o crescimento, que só aumentava,
de usuários mobile e como isso afetava (em 2010) as empresas que necessitavam que
seus sites tivessem uma boa performance e experiência também nos novos
dispositivos.

É importante entender que o design responsivo não é uma tecnologia separada, é um termo usado para descrever uma abordagem à web design, ou um conjunto de melhores práticas, usado para criar um layout que possa responder ao dispositivo que está sendo usado para visualizar o conteúdo.

O design responsivo sugere que o desenvolvimento e o design de uma aplicação web responda conforme as ações que o usuário fizer. Para isso é utilizado no desenvolvimento grades flexíveis e um layout que se adapte a diferentes tamanhos de tela. Um exemplo disso é que o usuário pode utilizar a mesma aplicação web em um desktop e mudar para um dispositivo 
mobile, e o layout se ajuste automaticamente não perdendo suas funcionalidades (KNIGHT, 2011).

\subsection{Frameworks de CSS}

O framework é um facilitador no desenvolvimento de diversas aplicações, e sem dúvida, sua utilização poupa tempo e custos para quem utiliza. Segundo Amorim (2017) frameworks constituem em uma estrutura pré-pronta de elementos para construir uma aplicação. No caso de CSS, os frameworks constituem bibliotecas que visam permitir a codificação do CSS de modo mais fácil e compatível com padrões de estilos, agregando uma série de opções já prontas para projetar uma página web, como se fosse um esqueleto, reduzindo o tempo gasto com o desenvolvimento (PAGANI, 2011).

\subsection{Bootstrap}

Bootstrap originalmente foi criado em meados de 2010 por Mark Otto e Jacob Thornton, um designer e um desenvolvedor no Twitter. O Bootstrap se tornou um dos mais populares frameworks front-end e projetos de código aberto no mundo (BOOTSTRAP, 2020a).

Para resolver um problema interno do Twitter, Otto e Thornton criaram o Bootstrap como solução para as inconsistências de código dentro da equipe. Antes não existia nenhum padrão para estrutura de código usada pela equipe. Cada engenheiro utilizava sua própria maneira de programar, com isso havia dificuldades na hora de juntar os módulos do projeto.

De acordo com (SILVA, 2019) a finalidade original do Bootstrap era incentivar o uso de uma única estrutura de código, nomenclatura de classes e dentre outras, pelas equipes de engenharia da empresa. A iniciativa foi bem sucedida, resultando em menos inconsistências e consequentemente maior rapidez nos projetos.

Antes de ser uma estrutura de código aberto, o Bootstrap era conhecido como Twitter Blueprint (Bootstrap). Em agosto de 2011, Bootstrap foi lançado publicamente no Github como um projeto de software livre.

"Projetado para qualquer um, em qualquer lugar o Bootstrap torna o desenvolvimento front-end web mais rápido e fácil. Ele é feito para pessoas de todos os níveis e dispositivos de qualquer forma ou tamanho" (BOOTSTRAP, 2015). 


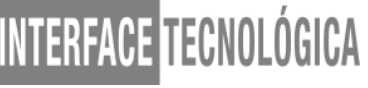

O Bootstrap conta com algumas características que o fazem ser o framework de css mais popular atualmente.

O seu código fonte utiliza o Sass, que é um pré-processador de CSS. Na versão do Bootstrap 3 o Less também era utilizado como pré-processador, mas na documentação da versão Alpha do Bootstrap 4 foi informado que houve uma migração do Less para Sass para os arquivos CSS de origem. Em relação a responsividade o Bootstrap é muito eficiente, utilizando medias queries de css, os projetos web se adaptam facilmente entre as telas de celulares, tablets e desktop. Além disso conta com ótimos recursos, sua documentação é muito rica em conteúdo e com um design bonito, dezenas de componentes HTML e CSS personalizados, e plugins jQuerys (BOOTSTRAP, 2015).

O Bootstrap 4 é a versão mais recente; com novos componentes, folha de estilo mais rápida, mais capacidade de resposta e suporta as versões mais recentes e estáveis de todos os principais navegadores e plataformas. No entanto, o Internet Explorer 9 e versões inferiores não são suportadas (W3SCHOOLS, 2019).

\subsection{Bulma}

Segundo site oficial, Bulma é uma estrutura CSS gratuita e de código aberto baseada no Flexbox, inspirado no Bootstrap e já tem uma compatibilidade bem grande entre os browsers (BULMA,2020).

Entre suas características, estão:

- $100 \%$ responsivo (mobile first)

- Totalmente CSS (não requer JavaScript)

- Modular (importe no seu projeto somente o que você precisa)

- Moderno (construído com Flexbox)

- Livre (open Source no GitHub)

O framework contém todos os elementos mais comuns, como: formulários, tabelas, botões. menus, títulos, notificações, barras de progresso, entre outras coisas (ESCUDELARIO, 2019). 


\section{PROCEDIMENTOS METODOLÓGICOS}

Para o desenvolvimento deste artigo foi realizado um levantamento bibliográfico através da pesquisa em sites especializados, artigos e livros sobre o assunto, nesse processo foram estudados ambos os frameworks de CSS propostos na comparação, para analisar quais seus principais recursos, suas diferenças, vantagens e desvantagens. O comparativo entre eles será apresentado no formato de tabela comparando os principais recursos e pontos de cada um dos frameworks.

\section{RESULTADOS E DISCUSSÕES}

\subsection{Semelhanças e diferenças}

Veja quais elementos da estrutura existem no Bootstrap e no Bulma na tabela abaixo.

Tabela 1 - Tabela de comparação

\begin{tabular}{|c|c|}
\hline Bootstrap & Bulma \\
\hline \multicolumn{2}{|c|}{ Sistema de grid } \\
\hline columns & row \\
\hline column & Col \\
\hline tile & - \\
\hline \multicolumn{2}{|c|}{ Formulários } \\
\hline field & - \\
\hline field is-grouped & button groups \\
\hline control & form-group \\
\hline label & label \\
\hline input & form-control \\
\hline textarea & form-control \\
\hline select & - \\
\hline checkbox & checkbox \\
\hline radio & radio \\
\hline file & - \\
\hline button is-static & form-control-static \\
\hline \multicolumn{2}{|c|}{ Elementos } \\
\hline box & - \\
\hline button & button \\
\hline content & - \\
\hline delete & close \\
\hline
\end{tabular}




\begin{tabular}{|c|c|}
\hline icon & glyphicon \\
\hline image & thumbnails \\
\hline image is-16by 9 & responsive embed \\
\hline notification & alerts \\
\hline progress & progress bars \\
\hline table & table \\
\hline tag & labels \\
\hline tag is-rounded & badges \\
\hline title & h1 .. h6 \\
\hline subtitle & h1 .. h6 \\
\hline- & lists \\
\hline- & caret \\
\hline & (continuação) \\
\hline Bootstrap & Bulma \\
\hline & \\
\hline breadcrumb & breadcrumbs \\
\hline card & - \\
\hline dropdown & dropdowns \\
\hline level & - \\
\hline media object & media object \\
\hline menu & - \\
\hline message & panels \\
\hline modal & modal \\
\hline navbar & navbar \\
\hline- & page header \\
\hline pagination & pagination \\
\hline panel & - \\
\hline tabs & navs \\
\hline- & wells \\
\hline \multicolumn{2}{|c|}{ Layout } \\
\hline container & container \\
\hline hero & jumbotron \\
\hline section & - \\
\hline footer & - \\
\hline
\end{tabular}

Fonte: Bulma (2020).

A tabela abaixo mostra características de cada framework.

Tabela 2 - Tabela geral de comparação

Bootstrap

O que o Bootstrap inclui:

\begin{tabular}{|l|r}
\multicolumn{1}{c|}{ Bootstrap } & Bulm \\
\hline O que o Bootstrap inclui: & O que o Bulma inclui:
\end{tabular}


- Estrutura básica com sistemas de grid

- Arquivo CSS

- Plugins JavaScript

- Tamanho minimizado de CSS: $68 \mathrm{~Kb}$. Gzip:12kb

- Número total de elementos: 49
- Comando is-*. has-*, usado para projetar arquivo. SCCS

- Sistema de grade próprio

- Não utiliza nenhum plugin jQuery e JavaScript

- Tamanho minimizado de CSS: 73kb. Gzip:10kb

- Número total de elementos: 53

(continuação)

\begin{tabular}{l|l}
\hline \multicolumn{1}{c|}{ Bootstrap } & \multicolumn{1}{c}{ Bulma } \\
\hline Grande comunidade: Considerando o tempo & Sistema de grade simples: Para criar uma \\
que existe, o Bootstrap tem uma comunidade & grade Bulma, você só precisa de um único \\
maior que a do Bulma. Como resultado, mais & container \\
ferramentas (como temas e plugins) estão & .columns para agrupar quantos itens .column \\
disponíveis. & desejar. \\
\hline
\end{tabular}

Acessibilidade: Bulma introduziu algum Não utiliza JavaScript: Ao focar apenas no suporte para acessibilidade, mas o Bootstrap CSS, a Bulma fornece uma solução leve que tem compatibilidade forte e difundida com as pode ser facilmente implementada em diretrizes da WCAG 2.0. qualquer contexto de desenvolvimento.

\begin{tabular}{l|l}
\hline Linguagens de programação: CSS, HTML, & Linguagens de programação: CSS, HTML, \\
JavaScript, Sass & Sass
\end{tabular}
Fonte: VYAS (2019).

\section{2 Vantagens e desvantagens do Bootstrap}

Vantagens: Bootstrap é muito utilizado pelos desenvolvedores. O que chama a atenção é sua facilidade de uso, e tem uma documentação muito rica e bem detalhada. $\mathrm{O}$ fato de ter um foco em mobile first é muito importante, pois segundo (LEONE, 2018) "indica-se que todos os 


\section{WNEREFACFETECNOLOGGCA}

sites devem ser responsivos para melhorar a experiência do usuário e trazer benefícios para rankeamento do Google."

Outro ponto importante e considerado uma vantagem segundo Gasparotto (2017), é sua capacidade de utilizar classes para esconder e mostrar elementos com facilidade. Além disso, também possibilita a utilização de classes para acessibilidade, para elementos como leitores de tela e navegação através do teclado.

Desvantagens: $O$ foco do Bootstrap não é ter um site visualmente diferenciado, a proposta principal é a experiência do usuário. Com isso há um excesso de padronizações, a maioria das aplicações que utilizam Bootstrap acabam tendo a mesma identidade.

Apesar de ter muitas opções de botões, painéis e ícones, ainda assim é comum você ver os mesmos elementos em diferentes portais. Isso acontece porque o framework é muito famoso. Pesquisas afirmam que cerca de 7 milhões de sites utilizam o Bootstrap (LEONE, 2018).

Alguns desenvolvedores também evitam a utilização do Bootstrap pois uma boa parte do código CSS presente no framework nunca será utilizado no projeto, isso consequentemente deixa o projeto mais pesado e lento (LEONE, 2018).

\subsection{Vantagens e desvantagens do Bulma}

Vantagens: "O Bulma é um framework CSS que vem ganhando muita notoriedade por ser moderno, responsivo, gratuito e modular" (SILVA, 2018), uma das vantagens é que ele é muito leve e fácil de se trabalhar, além disso um ponto muito interessante de ser modular, é que você consegue importar e utilizar somente o que precisa, tornando sua aplicação bem mais leve.

De acordo com (BULMA.2020), é adotado também o conceito de "first mobile", projetando a estrutura primeiramente para dispositivos moveis e segundo (DAITYARI, 2020) editor do site CODEINWP ter o foco somente em CSS, e não utilizar JavaScript também se torna uma vantagem. Por exemplo, fica mais leve, permite a escolha do framework de Javascript, ou até mesmo a utilização de Javascript "puro".

Desvantagens: Ser leve pode ser uma vantagem, porém não utilizar nenhum elemento javascript acaba se tornando uma desvantagem, pois no Bootstrap, existe vários plugins que facilitam muito o desenvolvimento, um exemplo é o plugin Modal, basicamente uma janela que abre sobre o conteúdo com o intuito de passar algum aviso ou informação para o usuário, 
também há o plugin Tooltips que é um pequeno pop-up que aparece quando o usuário direciona o ponteiro do mouse em cima um elemento (BOOTSTRAP, 2020b).

Uma outra desvantagem é que por ser uma nova estrutura, não tem uma comunidade muito grande, podemos ver que no GitHub o Bootstrap conta com 140 mil estrelas e é utilizado por um pouco mais de 1 milhão de repositórios, e o Bulma conta com um pouco mais de 39 mil estrelas e utilizado por 97 mil repositórios (GITHUB,2020).

\subsection{Algumas empresas que utilizam os Frameworks}

Tabela 4 - Empresas que utilizam Bootstrap ou Bulma

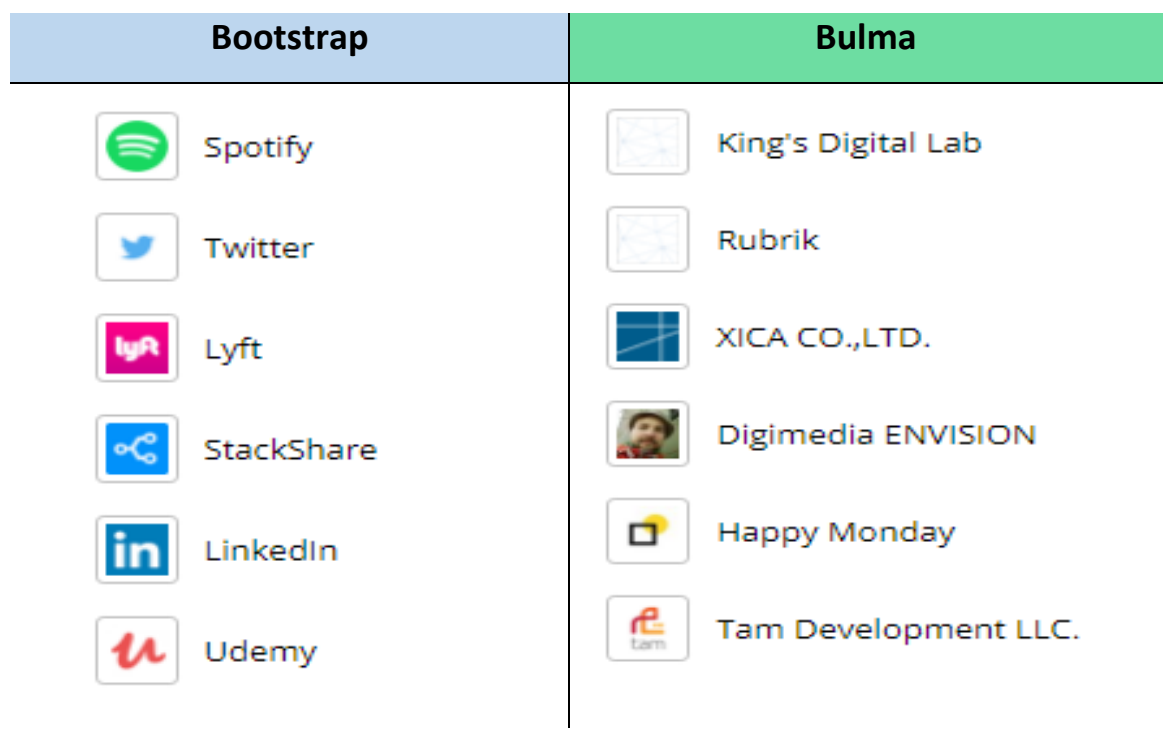

Fonte: stackshare (2020)

\section{CONSIDERAÇÕES FINAIS}

Os frameworks de CSS estão sendo utilizados cada vez mais pelos desenvolvedores, facilitam o desenvolvimento de qualquer aplicação web, são fáceis de usar e poupam tempo. Esses frameworks ganharam muito espaço no mercado, a maioria dos sites hoje em dia utiliza algum deles.

Devido a existência de vários frameworks no mercado, o desenvolvedor acaba tendo dificuldade na hora da escolha, com a análise comparativa realizada nesse trabalho, não visando decretar qual é o melhor framework entre o Bootstrap e o Bulma, e sim destacar que ambos são parecidos e tem pontos fortes e fracos, pretende-se ajudar nessa escolha do framework ideal. 
Um ponto forte do Bootstrap são os plugins que ele oferece, pois auxiliam e tornam o dia a dia do programador bem mais fácil, com várias bibliotecas prontas e disponíveis. Apesar do Bootstrap ser popular e mais utilizado, o Bulma tem um ótimo desempenho, é leve, fácil de aprender e muito moderno, o que acaba se tornando uma desvantagem é a falta elementos Javascript, sendo necessário o desenvolvimento do zero ou a utilização de um código adicional para situações como a de prover um banner rotativo por exemplo.

Devemos ressaltar, que esse artigo teve a finalidade de extrair o máximo de comparações, vantagens, pontos fortes e fracos de cada framework e auxiliar o desenvolvedor na escolha. Por fim, com base nos dados analisados, concluímos que cada framework tem suas características, a decisão de qual usar, depende exclusivamente das especificações que a aplicação à desenvolver irá exigir.

\section{REFERÊNCIAS}

AMORIM, Simone. Você precisa de um Framework CSS?: Leia primeiro e conte-me depois. 2017. Disponível em: https://medium.com/@simoneas02/voc\%C3\%AA-precisa-deum-framework-css-922c02ade6a5. Acesso em: 29 nov. 2019.

ARTY, David. Manual de Web Design Responsivo: Projete para Todos Dispositivos. São Paulo: [s. n.], 2015. 39 p. Disponível em: https://www.chiefofdesign.com.br/ebook-webdesign-responsivo/. Acesso em: 2 abr. 2020.

BOOTSTRAP. O Bootstrap é a estrutura HTML, CSS e JS mais popular do mundo para a criação de projetos responsivos para dispositivos móveis na Web. 2015. Disponível em: https://v4-alpha.getbootstrap.com/. Acesso em: 04 abr. 2020.

BOOTSTRAP. Sobre: visão geral. Visão geral. 2020a. Disponível em: https://getbootstrap.com.br/docs/4.1/about/overview/. Acesso em: 04 abr. 2020.

BOOTSTRAP. Documentação: componentes. Componentes. 2020b. Disponível em: https://getbootstrap.com.br/docs/4.1/components/. Acesso em: 04 abr. 2020.

BULMA. Bulma. 2020. Disponível em: https://bulma.io/. Acesso em: 03 abr. 2020.

BULMA. Coming from Bootstrap: See how Bulma is an alternative to Bootstrap. Disponível em: https://bulma.io/alternative-to-bootstrap/. Acesso em: 03 abr. 2020.

CONFIGR. O que são frameworks para desenvolvimento WEB. (Conheça os melhores para PHP, Python e Ruby). 31 julho 2019. Disponível em: $<$ https://configr.com/blog/frameworkspara-desenvolvimento-web/>. Acesso em: 20 nov. 2019. 
DEVMEDIA. Bate papo sobre Frameworks. Rio de Janeiro. Disponível em: https://www.devmedia.com.br/frameworks-uma-visao-inicial/37477. Acesso em: 20 nov. 2019.

DAITYARI, Shaumik. Bootstrap vs Foundation vs Bulma vs Semantic vs UIkit. 2020. Disponível em: https://www.codeinwp.com/blog/bootstrap-vs-foundation-vs-bulma-vssemantic-vs-uikit/. Acesso em: 04 abr. 2020.

ESCUDELARIO, Bruna de Freitas. Conhecendo o framework Bulma. 2019. Disponível em: https://imasters.com.br/css/conhecendo-o-framework-bulma. Acesso em: 03 abr. 2020.

GASPAROTTO, Henrique Machado. Bootstrap ou Materialize: Conheça as principais diferenças. 2017. Colaborador DEVMEDIA. Disponível em: https://www.devmedia.com.br/bootstrap-ou-materialize-conheca-as-principaisdiferencas/37830. Acesso em: 04 abr. 2020.

GITHUB. Twbs / bootstrap: The most popular html, css, and javascript framework for developing responsive, mobile first projects on the web. 2020. Disponível em: https://github.com/twbs/bootstrap. Acesso em: 05 abr. 2020.

GITHUB. Jgthms / bulma: modern css framework based on flexbox. Modern CSS framework based on Flexbox. 2020. Disponível em: https://github.com/jgthms/bulma. Acesso em: 05 abr. 2020.

JAIN, Nilesh. REVIEW OF DIFFERENT RESPONSIVE CSS FRONT-END FRAMEWORKS. Journal Of Global Research In Computer Science. Mandsaur, p. 1-6. 11 nov. 2014. Disponível em: http://www.jgrcs.info/index.php/jgrcs. Acesso em: 20 nov. 2019.

KNIGHT, Kayla. Responsive Web Design: What It Is and How To Use It. In: Smashing Magazine. 2011. Disponível em: https://www.smashingmagazine.com/2011/01/guidelinesfor-responsive-web-design/. Acesso em abril de 2020.

LEONE, Leonello de. Bootstrap: o que é, porque usar e como começar com o framework. 2018. Editor do BECODE. Disponível em: https://becode.com.br/bootstrap-o-que-e-porqueusar-e-como-comecar/. Acesso em: 04 abr. 2020.

MULLER, Nicolas. Framework, o que é e para que serve? 2008. Disponível em: https://www.oficinadanet.com.br/artigo/1294/framework_o_que_e_e_para_que_serve. Acesso em: 11 nov. 2019.

MARCOTTE, Ethan. 2010. Responsive Web Design. Disponível em: https://alistapart.com/article/responsive-web-design/, Acesso em: 2 de abril. 2020.

PAGANI, Talita. Quando utilizar (ou não) frameworks CSS: A utilização de frameworks HTML e/ou CSS ainda é um assunto que divide a opinião dos desenvolvedores. 2011. Disponível em: https://tableless.com.br/quando-utilizar-ou-nao-frameworks-css/, Acesso em: 29 nov. 2019. 
SILVA, Arthur de Almeida Pereira da. DESIGN RESPONSIVO: TÉCNICAS, FRAMEWORKS E FERRAMENTAS. 2014. 86 f. TCC (Graduação) - Curso de Sistemas de Informação, Centro de Ciências Exatas e Tecnologia, Universidade Federal do Estado do Rio de Janeiro (unirio), Rio de Janeiro, 2014.

STACKSHARE. JavaScript: lightweight, interpreted, object-oriented language with firstclass functions developer.mozilla.org. 2019. Disponível em:

https://www.stackshare.io/javascript. Acesso em: 05 abr. 2020.

STACKSHARE. Bootstrap vs Bulma. 2020. Disponível em:

https://stackshare.io/stackups/bootstrap-vs-bulma. Acesso em: 03 abr. 2020.

VYAS, Deep. Bootstrap vs. Bulma. 2019. Disponível em:

https://aspiresoftware.in/blog/bootstrap-vs-bulma/. Acesso em: 03 abr. 2020.

W3SCHOOLS. Bootstrap 4 Get Started. 2019. Disponível em:

https://www.w3schools.com/bootstrap4/bootstrap_get_started.asp. Acesso em: 03 abr. 2020. 